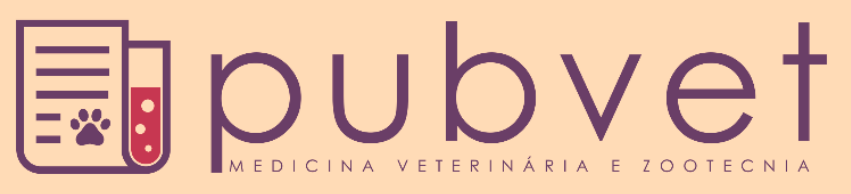

https://doi.org/10.31533/pubvet.v13n12a477.1-8

\title{
Influência da temperatura e umidade sobre o conforto térmico bovino em Barbalha, Ceará
}

\author{
Mirelle Tainá Vieira Lima ${ }^{1 *} \bullet$, José Valmir Feitosa ${ }^{2} \bullet$, Carlos Wagner Oliveira ${ }^{2} \bullet$, Antônio \\ Nélson Lima da $\operatorname{Costa}^{2} \theta$
}

${ }^{I}$ Mestranda na Universidade Federal do Cariri, Centro de Ciências Agrárias e da Biodiversidade. Crato -CE Brasil. ${ }^{2}$ Professor da Universidade Federal do Cariri, Centro de Ciências Agrárias e da Biodiversidade. Crato-CE Brasil. *Autor para correspondência, E-mail: mirellet.vieira@gmail.com

\begin{abstract}
Resumo. O presente trabalho teve por objetivo estimar a interferência de fatores climáticos sobre a produção de leite bovino no município de Barbalha, Ceará, utilizando os dados de temperatura, umidade relativa do ar para a obtenção do índice de temperatura e umidade UTI. Inicialmente, foi realizado o levantamento de dados meteorológicos de trinta anos (1986 a 2016) para o município de Barbalha, obtidos da estação meteorológica automática do Instituto Nacional de Meteorologia- INMET, localizada na Estação Experimental da Embrapa, no município de Barbalha-CE. Para os dados de temperatura de bulbo seco e de umidade relativa do ar foram feitas três coletas diárias $(0: 00 ; 12: 00$ e 18:00 h). O ITU verificado, em grande parte dos casos, encontra-se em nível de alerta, ou seja, é possível obter boa produtividade melhorando as instalações de produção, amenizando a interferência dos fatores de temperatura e umidade ambientais. Quanto aos horários estudados, as 21:00 h representou o melhor horário para o conforto térmico seguido das 9:00 e as 15:00 h apresentou os piores resultados de conforto térmico. Assim, o produtor deve atentar para os horários favoráveis ao manejo de seu rebanho, o que favorecerá o bemestar animal e, consequentemente, a produção leiteira.
\end{abstract}

Palavras chave: bem-estar, bioclimatologia, clima semiárido

\section{Influence of the temperature and humidity on the dairy production of Barbalha, Ceará, Brazil}

\begin{abstract}
This work aimed to estimate the influence of climatic factors over the cattle milk production in the municipality of Barbalha, ceará, through temperature and relative humidity data to get the temperature and humidity index - hti. Firstly, a thirty-year (1986 to 2016) meteorological data survey was carried out for the municipality of Barbalha-CE, obtained from the automatic meteorological station of the National Institute of Meteorology (INMET), located at the Embrapa Experimental Station in the same city. About the data of dry bulb temperature and relative air humidity three collections were done daily (at 0:00, 12:00 and 18:00 h). The HTI of Barbalha, for dairy cattle, finds in the mostly times a level of HTI that requires attention. Thus, it's possible to get better the production improving the elements related to Temperature and Humidity. About the studied hours, the 21:00 p.m. represented the best time for thermal comfort, followed by 9:00 a.m. and 3:00 p.m. presented the worst results of thermal comfort. Thus, the producer must pay attention to the favorable time for the cattle management, that will improve the animal welfare and, consequently, milk production.
\end{abstract}

Keywords: dairy cattle, semiarid climate, welfare 


\section{Influencia de la temperatura y la humedad en el confort térmico bovino en Barbalha, Ceará}

Resumen. El presente trabajo tuvo como objetivo estimar la interferencia de los factores climáticos en la producción de leche de vaca en el municipio de Barbalha-CE, utilizando la temperatura, la humedad relativa para obtener el índice de temperatura y humedad - UTI. Inicialmente, fueron recopilados los datos meteorológicos de treinta años (1986 a 2016) para el municipio de Barbalha, obtenidos de la estación meteorológica automática del Instituto Nacional de Meteorología-INMET, ubicado en la Estación Experimental Embrapa, en el municipio de Barbalha-CE. Para los datos de temperatura de bulbo seco y humedad relativa del aire, se tomaron tres muestras diarias $(0: 00 ; 12: 00$ y 18:00 h). La IU verificada en la mayoría de los casos se encuentra en el nivel de alerta, es decir, es posible obtener una buena productividad mejorando las instalaciones de producción, mitigando la interferencia de los factores de temperatura y humedad ambientales. En cuanto a las horas estudiadas, las 21:00 representaron el mejor momento para el confort térmico, seguido de las 9:00 y las 15:00 presentaron los peores resultados de confort térmico. Por lo tanto, el productor debe prestar atención a los tiempos favorables para el manejo de su rebaño, lo que favorecerá el bienestar animal y, en consecuencia, la producción de leche.

Palabras clave: bienestar, bioclimatología, clima semiárido

\section{Introdução}

O Brasil é privilegiado pela vasta disponibilidade de terras e clima favorável a produção da maioria dos alimentos. Neste contexto, a pecuária bovina no país é, de longe, a atividade econômica que ocupa a maior extensão de terras (ANUALPEC, 2019; Ferraz \& Felício, 2010).

Para a região semiárida do nordeste brasileiro, a atividade pecuária é de extrema importância, sobretudo a criação de ruminantes, a qual tem se constituído, ao longo do tempo, na atividade básica das populações rurais distribuídas nos 95 milhões de hectares da região semiárida nordestina (ANUALPEC, 2019; Nóbrega et al., 2011). Contudo, considerando que a maioria dos animais domésticos criados nos países tropicais descende de animais introduzidos pelos colonizadores europeus (Bianchini et al., 2006), o contraste de ambientes, a alta necessidade nutricional e o menor ritmo de alterações de manejo, geraram animais pouco adaptados e com grau de bem-estar bastante comprometido (Bond et al., 2012). Nesse contexto, o estudo da bioclimatologia busca entender como o animal interage e responde às diferentes condições de ambiente, sendo uma ferramenta para definir as condições de manejo ideais de produção (Berbigier, 1988; Pereira, 2005; Silva, 2000).

Vários índices foram desenvolvidos, a fim de avaliar o nível de conforto térmico dos animais em relação ao ambiente. $\mathrm{O}$ mais utilizado foi o índice de temperatura e umidade (ITU), primeiramente desenvolvido por Thom (1959) e adaptado posteriormente por diversos autores, para a avaliação de animais de produção, cuja fórmula relaciona os efeitos da temperatura e umidade relativa do ar sobre o bem-estar animal.

Dessa forma, o presente trabalho tem por objetivo avaliar a adequação climática do município de Barbalha para a atividade leiteira, tendo como parâmetro o índice de temperatura e umidade (ITU) ao longo do ano para, com isto, verificar os períodos (seco, chuvoso ou de transição) e os horários mais favoráveis à produção.

\section{Material e métodos}

O município de Barbalha está situado nas coordenadas $7^{\circ} 18^{\prime} 40^{\prime \prime}$ e $3918^{\prime}$ 15" Sul (Figura 1). O clima é Tropical Semiárido, a temperatura média de $24^{\circ}$ a $26^{\circ} \mathrm{C}$, e pluviosidade média anual de 1.153 $\mathrm{mm} /$ ano com período chuvoso concentrado de janeiro a abril (IPECE, 2017). Possui Classificação climática Thornthwaite B1 W W A' a' e Köppen Aw (Vieira et al., 2017).

Inicialmente, foi realizado o levantamento de dados meteorológicos no período de trinta anos (1986 à 2016) para o município de Barbalha, obtidos da estação meteorológica automática do Instituto 
Nacional de Meteorologia- INMET, localizada na Estação Experimental da Embrapa, no município de Barbalha-CE, com as seguintes coordenadas geográficas: latitude $07^{\circ} 19^{\prime} \mathrm{S}$, longitude $39^{\circ} 18^{\prime} \mathrm{W}$ e altitude de $415,74 \mathrm{~m}$. Os dados de temperatura de bulbo seco e umidade relativa do ar correspondem a três coletas diárias (0:00 12:00 e 18:00 h), os quais foram tabulados em planilha Excel e processados estatisticamente por meio do programa SAS (2004).

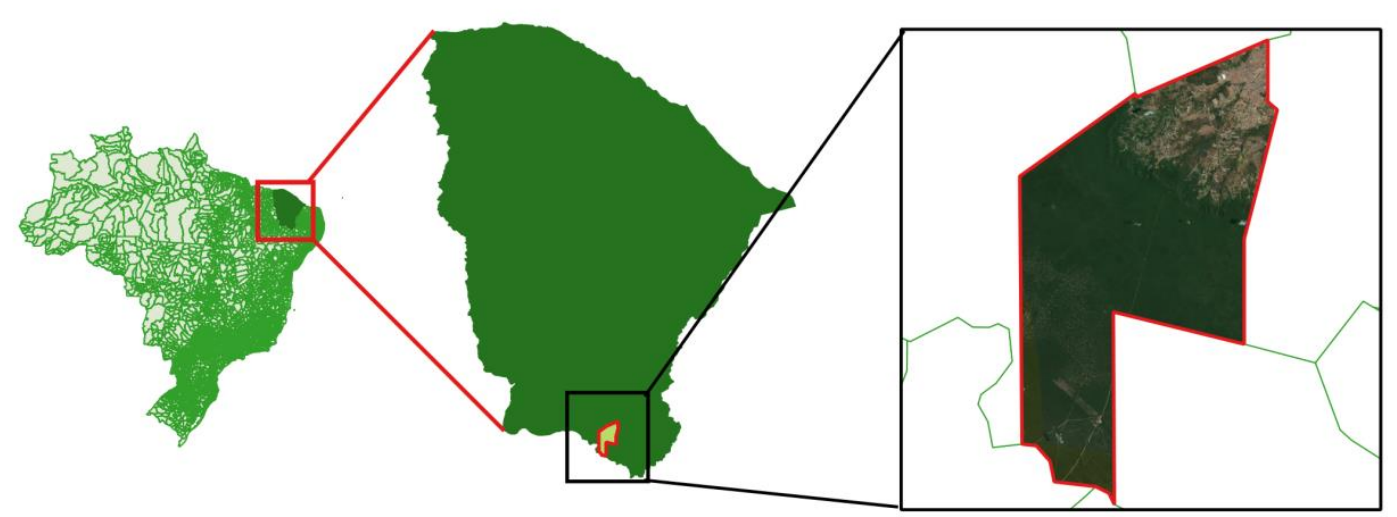

Figura 1. Mapa de localização do município de Barbalha - CE. Fonte: Elaboração dos autores.

O ITU corresponde a um modelo matemático desenvolvido para analisar o nível de bem-estar de vacas em lactação, e constitui um método eficaz e prático, largamente utilizado em todo o mundo para estudos de bioclimatologia. Para o cálculo do ITU são utilizadas a temperatura ambiente (TA) e a umidade relativa do ar (UR). Esses dois fatores são os principais elementos climáticos que influenciam o estresse térmico em bovinos leiteiros, de acordo com a equação utilizada e adaptada por Buffington et al. $(1981):$ ITU $=(0,8 * \mathrm{TA}+(\mathrm{UR} \%) *(\mathrm{TA}-14,4)+46,4)$. Onde, TA: Temperatura do ambiente, ${ }^{\circ} \mathrm{C}$; UR: Umidade relativa do ar (\%).

Ponderando os dados da literatura acerca da classificação de ITU para bovinos, neste trabalho foi assumida a seguinte classificação, conforme Pires \& Campos (2004). Inferior a 70: normalmente (os animais encontram-se numa faixa de temperatura e umidade ideal para seu desempenho produtivo); Maior que ou igual a 70 e menor que ou igual a 72: alerta (as condições climáticas estão no limite para o bom desempenho produtivo); Maior que 72 e menor que ou igual a 78: alerta, e acima do índice crítico para a produção de leite (nesta faixa, o desempenho produtivo está comprometido); Maior que 78 e menor que ou igual a 84: perigo (todas as funções orgânicas dos animais estão comprometidas); Superior a 84: emergência (providências urgentes devem ser tomadas).

\section{Resultados e discussão}

O gráfico 1 apresenta a temperatura média mensal no município de Barbalha-CE, entre os anos de 1987 e 2016. As médias, moda e mediana das temperaturas mensais são representadas pelas faixas pretas no gráfico e variaram de aproximadamente 25 a $28^{\circ} \mathrm{C}$. Os meses estão representados no eixo horizontal de 1 a 12 , onde os mais quentes concentraram-se de setembro a janeiro e no restante do ano, especificamente entre fevereiro e agosto, as temperaturas médias mantiveram-se na faixa de $25^{\circ} \mathrm{C}$, não ultrapassando a média de $26^{\circ} \mathrm{C}$. Os meses entre fevereiro e maio foram os que apresentaram maior estabilidade entre as médias. Os meses mais quentes foram outubro e novembro, ambos com a média de $28,30^{\circ} \mathrm{C}$.

As linhas verticais em cada extremidade representam os valores limite máximo e mínimo alcançados em cada mês, que para temperatura (Tbs) variaram de 14,8 a $37,8^{\circ} \mathrm{C}$. Abaixo da faixa central que indica a média, em cada bloco, representa a concentração do primeiro quartil, ou seja, $25 \%$ das ocorrências. Acima da mediana e média encontra-se o terceiro quartil que representa a concentração de $75 \%$ dos dados analisados. Pode-se analisar separadamente no Gráfico 2 o comportamento normal da umidade relativa do ar (UR\%) para cada mês.

A média mensal de umidade relativa do ar (\%) variou de 52 a 82 , apresentando variação ao longo do ano, sendo crescente de janeiro a março e apresentando queda gradual e constante até o mês de setembro, 
voltando a aumentar levemente após outubro. Os maiores níveis de umidade estão presentes na faixa entre janeiro e maio. A umidade média encontra-se dentro dos limites impostos pela literatura para o bem-estar animal, que está geralmente entre $50-90 \%$.

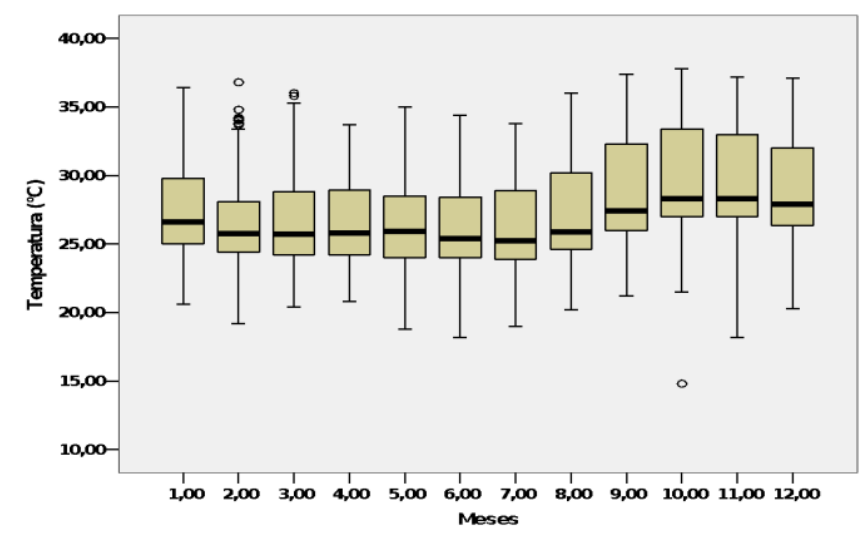

Gráfico 1. Diagrama de caixa das temperaturas médias mensais, de janeiro a dezembro, no município de Barbalha-CE, obtidas na base de dados do INMET de 1987 a 2016.

Pelo cálculo de ITU podem-se integralizar os valores de temperatura do bulbo seco $\left({ }^{\circ} \mathrm{C}\right)$ e umidade relativa do ar (\%), correlacionando os fatores para estimar o nível de conforto térmico animal, dessa forma, os mesmos dados foram submetidos à fórmula e obteve-se o resultado exposto no Gráfico 3.

As médias de ITU variaram entre 73,74 e 77,82, respectivamente nos meses de julho e novembro, com relativa estabilidade na maioria dos meses do ano. Em média, de janeiro a abril, o ITU esteve na faixa de 76, apresentando queda progressiva até julho e aumentando novamente a partir do mês de agosto. A média de ITU em todos os meses do ano permaneceu na faixa maior que 72 e menor ou igual a 78, o que denota estado de alerta para o rebanho, visto que esta faixa está acima do índice crítico para a produção de leite, onde o desempenho produtivo encontra-se comprometido. A umidade relativa do ar mínima e máxima variou respectivamente de 14 a $100 \%$.

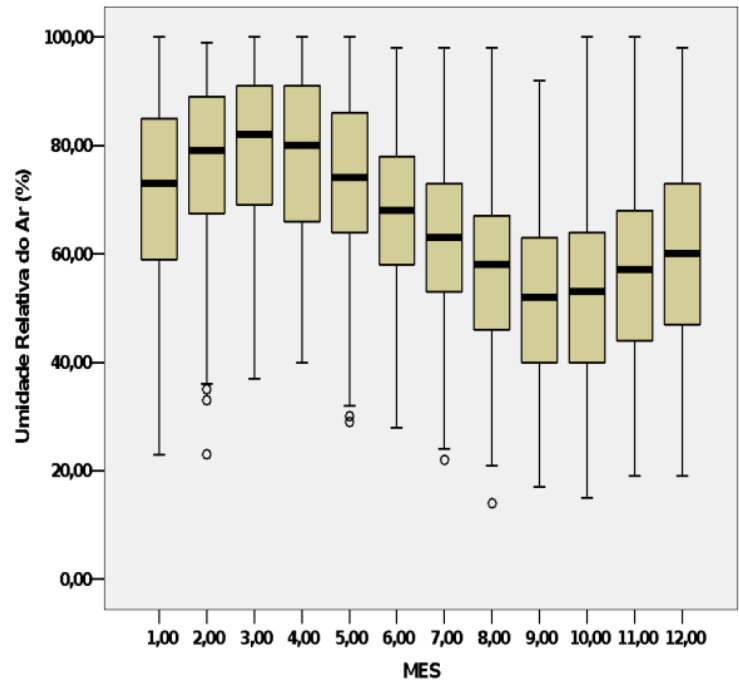

Gráfico 2. Diagrama de caixa das médias de umidade relativa do ar (\%) mensais, de janeiro a dezembro, no município de Barbalha-CE, obtidas na base de dados do INMET de 1987 a 2016.

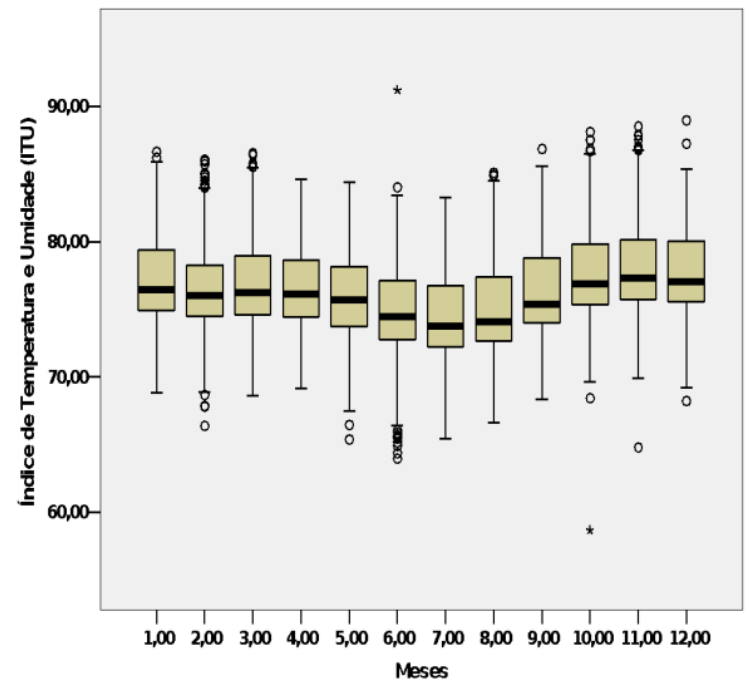

Gráfico 3. Diagrama de caixa do ITU mensal, de janeiro a dezembro, no município de Barbalha - CE, com base nos dados de temperatura e umidade médias, obtidas na base de dados do INMET de 1987 a 2016.

Armstrong (1994) classificou o estresse térmico de acordo com a variação de ITU em ameno ou brando (72 a 78), moderado (79 a 88) e severo (89 a 98). Autores como Johnson (1980) e Bertoncelli et al. (2013) asseguram que vacas Holandesas apresentam situação de estresse e queda de produção de leite com valores 
de ITU acima de 72. Igono et al. (1992) consideram estressante, para vacas com alta produção de leite, um ITU acima de 76. Em contrapartida, dados mais atualizados em pesquisas realizadas por Zimbelman et al. (2009) e Collier et al. (2012) mostraram que reflexos negativos na produtividade em raças europeias de alta produção, como o declínio da produção de leite em vacas leiteiras, podem ser observados em ITU acima de 68. Para uma melhor observação dos resultados acerca de média e desvio padrão em cada aspecto analisado e meses do ano, encontram-se dispostos na Tabela 1.

Tabela 1. Valores médios $(X)$ e desvio padrão (S) para os dados de temperatura do bulbo seco (TBS) umidade relativa do ar (UR\%) e índice de temperatura e umidade (ITU) para cada um dos meses do ano, no município de Barbalha-CE (1987-2016).

\begin{tabular}{lcccccc}
\hline \multirow{2}{*}{ Meses } & \multicolumn{2}{c}{ TBS } & \multicolumn{2}{c}{ UR (\%) } & \multicolumn{2}{c}{ ITU } \\
\cline { 2 - 7 } & $X$ & $\mathrm{~S}$ & \multicolumn{1}{c}{$X$} & $\mathrm{~S}$ & $\mathrm{~S}$ & 3,08 \\
Janeiro & 26,60 & 3,36 & 71,33 & 11,15 & 76,41 & 2,83 \\
Fevereiro & 25,75 & 3,79 & 79,00 & 14,17 & 76,00 & 9,06 \\
Março & 25,70 & 2,90 & 82,00 & 13,91 & 76,22 & 8,43 \\
Abril & 25,80 & 2,82 & 80,00 & 14,20 & 76,12 & 3,10 \\
Maio & 25,90 & 2,94 & 74,00 & 14,47 & 75,66 & 3,14 \\
Junho & 25,40 & 2,99 & 68,00 & 14,26 & 74,41 & 3,06 \\
Julho & 25,20 & 3,02 & 63,00 & 14,43 & 73,74 & 3,10 \\
Agosto & 25,85 & 3,27 & 57,01 & 14,27 & 74,04 & 3,11 \\
Setembro & 27,40 & 3,51 & 52,00 & 14,49 & 75,34 & 3,11 \\
Outubro & 28,30 & 3,51 & 53,00 & 16,37 & 77,48 & 3,14 \\
Novembro & 28,30 & 3,50 & 57,00 & 16,32 & 77,82 & 3,05 \\
Dezembro & 27,90 & 3,50 & 60,00 & 17,01 & 77,59 & \\
\hline
\end{tabular}

Sabendo-se que o desvio padrão é uma medida de dispersão em torno de uma média e expressa a homogeneidade dos dados, quanto maior o desvio padrão maior será a dispersão e distância dos dados em relação à média. Pode-se, assim, avaliar que os dados de temperatura do bulbo seco e índice de temperatura e umidade apresentaram pouca variação em torno da média, enquanto que a umidade relativa do ar apresentou-se mais dispersa.

As variações médias expressas para cada mês, no entanto, sofreram bastante discrepância em relação ao horário do dia. Da base do INMET foram coletados os dados horários de 21:00, 09:00 e 15:00 h e analisados separadamente conforme a Tabela 2.

Tabela 2. Valores médios $(X)$ e desvio padrão (S) para os dados de temperatura do bulbo seco (TBS) umidade relativa do ar (UR\%) e índice de temperatura e umidade (ITU) para cada um dos horários estudados, no município de Barbalha-CE (1987-2016).

\begin{tabular}{lcccccc}
\hline \multirow{2}{*}{ Meses } & \multicolumn{2}{c}{ TBS } & \multicolumn{3}{c}{ UR (\%) } & \multicolumn{2}{c}{ ITU } \\
\cline { 2 - 7 } & $X$ & S & $X$ & S & $X$ & S \\
\hline $21: 00$ & 24,90 & 2,13 & 76,00 & 16,59 & 74,27 & 2,46 \\
$09: 00$ & 25,90 & 1,56 & 71,00 & 13,02 & 75,15 & 1,77 \\
$15: 00$ & 31,20 & 6,57 & 51,00 & 15,41 & 79,80 & 2,22 \\
\hline
\end{tabular}

Às 21:00 h, os valores de Tbs, UR\% e ITU foram os mais favoráveis, seguido dos resultados obtidos às 9:00 h. Entretanto, média de temperatura do bulbo seco foram superiores às 15:00 h, contrastando com o menor índice de umidade relativa do ar no mesmo horário, que resultou no ITU superior em relação aos demais. Da mesma forma que na tabela anterior, a umidade relativa do ar foi a que sofreu maior variação de desvio padrão.

Foram analisadas a relação do número de ocorrências de cada faixa de ITU. Para tal, em cada mês as ocorrências foram separadas por faixas, posteriormente foram quantificadas e feita a sua correlação com o número total de ocorrências, obtendo assim, a porcentagem (Tabela 3).

Em todos os meses do ano, o ITU esteve concentrado na maior parte do tempo na faixa maior que 72 e menor do que ou igual a 78, seguido em quantidade, pela faixa maior do que 78 e menor do que ou 
igual a 84, exceto pelo mês de julho, onde a ocorrência de faixa de ITU maior ou igual a 70 e menor ou igual a 72 apresentou-se como a segunda maior em porcentagem de eventos. Em julho não foram observados casos de ITU acima de 84. Os dados percentuais, analisados em função dos horários, seguem na Tabela 4.

Tabela 3. Porcentagem média do número de horas mensais de ocorrência de ITU médio dentro dos intervalos estudados, no município de Barbalha - CE (1987-2016).

\begin{tabular}{lccccc}
\hline & \multicolumn{5}{c}{ Intervalos de ITU med. (\%) } \\
\cline { 2 - 6 } & ITU <70 & $70 \geq$ ITU $\leq 72$ & $72>$ ITU $\leq 78$ & $78>$ ITU $\leq 84$ & ITU $>84$ \\
\hline Janeiro & 0,76 & 2,97 & 62,59 & 32,54 & 1,14 \\
Fevereiro & 1,48 & 4,25 & 67,88 & 25,85 & 0,55 \\
Março & 1,96 & 4,47 & 63,98 & 28,23 & 1,36 \\
Abril & 1,33 & 3,87 & 65,57 & 28,94 & 0,29 \\
Maio & 2,49 & 6,92 & 64,70 & 25,73 & 0,16 \\
Junho & 6,02 & 10,86 & 66,12 & 16,94 & 0,06 \\
Julho & 5,54 & 17,82 & 61,76 & 14,88 & 0,00 \\
Agosto & 2,45 & 13,56 & 63,48 & 20,17 & 0,34 \\
Setembro & 1,33 & 4,10 & 64,39 & 29,02 & 1,16 \\
Outubro & 0,32 & 1,41 & 59,44 & 35,37 & 3,46 \\
Novembro & 1,02 & 1,50 & 55,54 & 38,51 & 3,43 \\
Dezembro & 1,03 & 2,11 & 58,85 & 35,60 & 2,42 \\
\hline
\end{tabular}

Nos horários de 21:00 e 9:00 h não foram observados valores de ITU superiores à 84 e, na maior parte do tempo, o ITU nesses horários esteve concentrado entre 78 e 84. Às 21:00 h há maior ocorrência de ITU inferior a 72 e menor concentração por faixa. No entanto, no período de 15:00 h, o ITU maior que 84 representou 3,79\% das ocorrências, além de ter apresentado maior incidência de ITU entre 78$84(75,74 \%)$. O ITU superior, às 15:00 h, provavelmente, deve-se a maior exposição solar, comum ao período da tarde. Os efeitos do clima vão amenizando com o decorrer da tarde, mas, ainda oferecendo desconforto pelo fato do solo absorver radiação durante o dia e nas primeiras horas da noite liberar esse calor em forma de vapor.

De acordo com Wanzeler et al. (2016), durante o dia ocorre o aquecimento da superfície, dando origem a um fluxo de calor para o interior do solo, elevando o acúmulo de energia e, consequentemente, a sua temperatura. Entretanto, à noite, em função do resfriamento da superfície, por emissão de radiação terrestre, há diminuição da temperatura nas camadas mais superficiais, que inverte o sentido do fluxo de calor, tornando-o ascendente e retornando o calor armazenado para a superfície.

Tabela 4. Avaliação horária da ocorrência dos intervalos de ITU dados em porcentagem, no município de Barbalha-CE (1987-2016).

\begin{tabular}{lccccc}
\hline \multirow{2}{*}{ Horário } & \multicolumn{5}{c}{ Intervalos de ITU méd \% } \\
\cline { 2 - 6 } & ITU $<70$ & $70 \geq$ ITU $\leq 72$ & $72>$ ITU $\leq 78$ & $78>$ ITU $\leq 84$ & ITU $>84$ \\
\hline $21: 00$ & 4,77 & 12,67 & 75,99 & 6,57 & 0,00 \\
$09: 00$ & 1,44 & 5,10 & 90,36 & 3,10 & 0,00 \\
$15: 00$ & 0,06 & 0,30 & 20,12 & 75,74 & 3,79 \\
\hline
\end{tabular}

Dessa forma, observa-se que é possível produzir leite satisfatoriamente em ambientes de clima semiárido com eficiência, o que requer uma boa gestão de recursos e melhorias no ambiente onde se encontram os animais. Para o incremento na produção da bovinocultura leiteira, nas mesmas condições deste trabalho, faz-se necessário promover a climatização do ambiente, através do seu resfriamento com a utilização de ventiladores, aspersores, exaustores e até tecnologias mais avançadas como o túnel de vento para atingir níveis satisfatórios de bem-estar animal e, consequentemente, produtividade.

\section{Conclusões}

O ITU médio, no município de Barbalha, relacionado à atividade da bovinocultura leiteira, encontrase, na maioria dos meses, em nível de alerta (72 a 78). O horário de 21:00 hs mostrou-se o mais adequado para a atividade leiteira e o horário das 15:00 hs, foi o mais inadequado. 


\section{Agradecimentos}

Agradecemos ao apoio concedido pela Coordenação de Aperfeiçoamento de Pessoal de Nível Superior (CAPES) pelo auxílio financeiro obtido através da concessão de bolsas de pesquisa.

\section{Referências bibliográficas}

ANUALPEC. (2019). Anuário da Pecuária Brasileira (20th ed. Vol. 1). São Paulo, São Paulo, Brasil: Instituto FNP.

Armstrong, D. V. (1994). Heat stress interaction with shade and cooling. Journal of Dairy Science, 77(7):2044-2050. doi: http://dx.doi.org/10.3168/jds.S0022-0302(94)77149-6.

Berbigier, P. (1988). Bioclimatologie des ruminants domestiques en zone tropicale (Vol. 1). Paris: INRA.

Bertoncelli, P., Martin, T. N., Ziech, M. F., Paris, W. \& Cella, S. (2013). Conforto térmico alterando a produção leiteira. Enciclopédia Biosfera, 9(17):762-777.

Bianchini, E., McManus, C., Lucci, C. M., Fernandes, M. C. B., Prescott, E., Mariante, A. S. \& Egito, A. A. (2006). Características corporais associadas com a adaptação ao calor em bovinos naturalizados brasileiros. Pesquisa Agropecuaria Brasileira, 41(9):1443-1448.

Bond, G., B., Almeida, R., Ostrensky, A. \& Molento, C. F. M. (2012). Métodos e pontos críticos de bem-estar de bovinos leiteiros. Ciência Rural, 421286-1293. doi: https://doi.org/10.1590/S010384782012005000044.

Buffington, D. E., Collazo-Arocho, A., Canton, G. H. \& Pitt, D. (1981). Black globe-humidity index (BGHI) as comfort equation for dairy cows. Transactions of the ASAE [American Society of Agricultural Engineers], 24(3):711-714.

Collier, R. J., Hall, L. W., Rungruang, S. \& Zimbleman, R. B. (2012). Quantifying heat stress and its impact on metabolism and performance. Paper presented at the Proceedings of the Florida Ruminant Nutrition Symposium, University of Arizona, USA.

Ferraz, J. B. S. \& Felício, P. E. (2010). Production systems - An example from Brazil. Meat Science, 84(2):238-243. doi: http://dx.doi.org/10.1016/j.meatsci.2009.06.006.

Igono, M. O., Bjotvedt, G. \& Sanford-Crane, H. T. (1992). Environmental profile and critical temperature effects on milk production of Holstein cows in desert climate. International Journal of Biometeorology, 36(2):77-87.

IPECE - Instituto de Pesquisa e Estratégia Econômica do Ceará [Internet]. (2017). Disponível em: http://www.ipece.ce.gov.br/index.php/perfil-municipal-2017. Acesso em: maio de 2018.

Johnson, H. D. (1980). Environmental management of cattle to minimize the stress of climatic change. International Journal of Biometeorology, 2465-78.

Nóbrega, G. H., Silva, E. M. N., Souza, B. B. \& Mangueira, J. M. (2011). A produção animal sob a influência do ambiente nas condições do semiárido nordestino. Revista Verde de Agroecologia e Desenvolvimento Sustentável, 6(1):67-73.

Pereira, J. (2005). Fundamentos de bioclimatologia aplicados à produção animal. Belo Horizonte: FEPMVZ.

Pires, M. D. F. A. \& Campos, A. T. (2004). Modificações ambientais para reduzir o estresse calórico em gado de leite. Embrapa Gado de Leite.

SAS. (2004). SAS/STAT User guide, Version 9.1.2. Cary, NC, USA: SAS Institute Inc.

Silva, R. G. (2000). Introdução à bioclimatologia animal. São Paulo, Brasil: Nobel.

Thom, E. C. (1959). The discomfort index. Weatherwise, 12(1):57-70.

Vieira, M. T., Meireles, A. C. M., Oliveira, C. W. \& Nascimento, M. T. B. (2017). Koppen-Geiger and Thornthwaite climatic classification for the metropolitan region of the Cariri, Ceará. Revista Geama, 3(3):136-143. 
Wanzeler, R. T. S., Costa, J. d. P. R. \& Santos, C. A. (2016). Variabilidade horária do perfil de temperatura do solo em um pomar de mangueiras (Mangifera indica 1.), na localidade de Cuiarana, Salinópolis-PA. Estação Científica (UNIFAP), 6(1):117-124.

Zimbelman, R. B., Rhoads, R. P., Rhoads, M. L., Duff, G. C., Baumgard, L. H. \& Collier, R. J. (2009). A re-evaluation of the impact of temperature humidity index (THI) and black globe humidity index (BGHI) on milk production in high producing dairy cows. Paper presented at the Proceedings of the Southwest Nutrition Conference, Arizona, USA.

Recebido: 26 de outubro, 2019.

Aprovado: 27 de novembro, 2019.

Publicado: 24 de janeiro, 2020.

Licenciamento: Este artigo é publicado na modalidade Acesso Aberto sob a licença Creative Commons Atribuição 4.0 (CC-BY 4.0), a qual permite uso irrestrito, distribuição, reprodução em qualquer meio, desde que o autor e a fonte sejam devidamente creditados 